\title{
Image Reconstruction by Prioritized Incremental Normalized Convolution
}

\author{
Anders Landström, Frida Nellros, Håkan Jonsson, and Matthew Thurley \\ Department of Computer Science, Electrical and Space Engineering, \\ Luleå University of Technology, 97187 Luleå, Sweden \\ \{andlan,frinel, hj, mjt\}@ltu.se \\ http://www.csee.ltu.se
}

\begin{abstract}
A priority-based method for pixel reconstruction and incremental hole filling in incomplete images and 3D surface data is presented. The method is primarily intended for reconstruction of occluded areas in 3D surfaces and makes use of a novel prioritizing scheme, based on a pixelwise defined confidence measure, that determines the order in which pixels are iteratively reconstructed. The actual reconstruction of individual pixels is performed by interpolation using normalized convolution.

The presented approach has been applied to the problem of reconstructing 3D surface data of a rock pile as well as randomly sampled image data. It is concluded that the method is not optimal in the latter case, but the results show an improvement to ordinary normalized convolution when applied to the rock data and are in this case comparable to those obtained from normalized convolution using adaptive neighborhood sizes.
\end{abstract}

Keywords: image reconstruction, hole filling, normalized convolution.

\section{Introduction}

There are many ways in which an image can be incomplete. Image sensors can be faulty, 3D surface image data can contain areas of missing data due to surface reflectance properties and occlusion [9] or image pixels can be lost or distorted during transmission of data.

As an example of missing data, including sensor occlusion, consider Fig. 6 (on page 183) showing two grayscale images depicting 3D surface data of a rock pile where missing pixels are marked in black. The image to the right shows the rows of range data as measured by a structured lighting sensor [9]. To simplify later analysis of the measurements, we consider how these missing pixels can be reconstructed.

While many techniques deal with reconstruction of randomly missing pixels [3, 6, 7, 8, there is also a potential benefit from being able to reconstruct missing regions within an image. This particular kind of image reconstruction is known as hole filling and can be summarized into three broad categories. 
Inpainting describes the technique where an artist reconstructs missing sections in a painting. This process can be formalized into solvable mathematical problems with the aim of producing visually pleasing images [1, 2,

Geometric methods are typically used when image data comprises a surface of $3 \mathrm{D}$ points, or it is appropriate to represent image pixels in this way. Points are triangulated into a mesh, upon which the reconstruction is based. Holes appear as non-triangulated parts of the mesh. These methods fill each hole by computing a suitable patch that fits in seamlessly within the close proximity of the hole. The patch is then sampled to get values for the missing points [5].

Kernel regression methods are commonly used for reconstruction of images based on sparse sets of irregularly sampled pixels, but can also be applied to whole regions of missing pixels. These methods are based on a foundation of linear algebra and use basis expansions of local neighborhoods to improve or fill in the data of a pixel [8]. The neighborhoods are weighted by an applicability function so the key point in these methods is how to choose the neighborhood size, shape and applicability. More recently, methods that adapt the neighborhood size according to the density of sampled points in the neighborhood and the shape of the applicability function to shapes of edges surrounding the neighborhood have been presented [7, 8].

In this paper we present a Prioritized Incremental algorithm using Normalized Convolution (PINC) for reconstruction of missing regions in incomplete images and 3D surface data. Specifically, when applied to surface data of piled particles (e.g. rocks) the presented method seeks to reconstruct the data in a way that preserves local topological variation and particle distinctness. The method makes use of a novel prioritizing scheme, based on a pixelwise defined confidence measure, that determines the order in which pixels are iteratively reconstructed. The actual reconstruction is performed by interpolation using the kernel regression method known as normalized convolution [6].

\section{Method}

At any time during the reconstruction process for an image, every pixel can be sorted into one of the three following classes:

1. Valid pixels, where the original image contains data.

2. Unfilled pixels; non-valid pixels where no value has been assigned.

3. Filled pixels; non-valid pixels that have been assigned an interpolated value.

The presented method reconstructs holes (regions of unfilled pixels) in a data set by interpolation of unfilled pixels in an order such that those with more "reliable data" in their proximity are processed before those with less. In order to achieve this reconstruction, a prioritizing strategy needs to be defined. Such a prioritization can be achieved by using a measure of the "validity" of the neighbors of an unfilled pixel. 
Let $d(\boldsymbol{x})$ denote the 2D Euclidean distance from the pixel $\boldsymbol{x}$ to the closest pixel containing valid data. A pixelwise confidence measure can then be defined as

$$
w_{c}(\boldsymbol{x})=\left\{\begin{array}{cl}
1 & \text { if } \boldsymbol{x} \text { contains valid data } \\
0 & \text { if } \boldsymbol{x} \text { contains unfilled data } \\
\frac{1}{d(\boldsymbol{x})+1} & \text { if } \boldsymbol{x} \text { contains filled data }
\end{array}\right.
$$

As unfilled pixels are filled, their confidence measure is changed according to (11). Pixel confidence values for filled data thus monotonically decrease from 1 at the border of valid data towards 0 in the unfilled pixels.

Consider an unfilled pixel $\boldsymbol{x}$ with a surrounding $n \times n$ neighborhood $N_{\boldsymbol{x}, n}$ containing pixels $\boldsymbol{x}_{N, 1}, \boldsymbol{x}_{N, 2}, \ldots, \boldsymbol{x}_{N, n^{2}}$. By summing up the confidence of the pixels in $N_{\boldsymbol{x}, 3}$, a priority measure $p(\boldsymbol{x})$ based on the confidence in the immediate neighborhood of $\boldsymbol{x}$ can then be defined as

$$
p(\boldsymbol{x})=\sum_{k=1}^{9} w_{c}\left(\boldsymbol{x}_{N, k}\right) .
$$

Since $d(\boldsymbol{x}) \geq 0,0 \leq w_{c}(\boldsymbol{x}) \leq 1$ and $0 \leq p(\boldsymbol{x}) \leq 8$. For example, if $\boldsymbol{x}$ is a unfilled pixel entirely surrounded by valid data, $p(\boldsymbol{x})$ would be 8 and that pixel should consequently be filled in before a pixel with fewer valid neighbors. The range of $p(\boldsymbol{x})$ depends on $w_{c}(\boldsymbol{x})$, and will thus change if another confidence measure is selected. The idea is that $w_{c}$ should be chosen so that holes are filled inwards from their perimeters.

The order of reconstruction is thus determined by prioritizing pixels using the defined confidence measure. Unfilled pixels of equal priority are processed in the same step. Knowledge of data set restrictions can be included where values are known to be within a certain interval. This is accomplished by truncating each assigned value within the interval limits directly after the interpolation step.

The PINC algorithm is a combination of a strategy for selecting the order for filling-in missing data and a method for assigning values to the unfilled pixels. It can be summarized as follows:

1

2

3

4

5

6

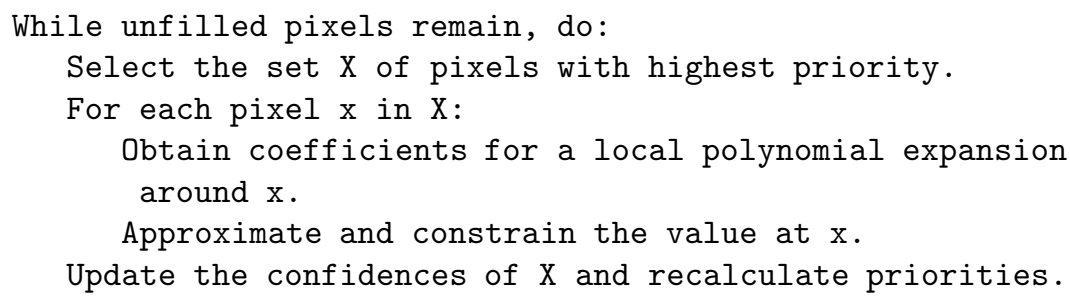

\subsection{Local Polynomial Expansion: Assigning Values}

Let $\boldsymbol{x}$ denote an unfilled pixel and $f$ the pixelwise signal values of the data set. The value of $\boldsymbol{x}, f(\boldsymbol{x})$, can be approximated by the constant-term coefficient for a best-fit local expansion in a selected basis (for instance, consider the constant term in a Taylor expansion). In this work a polynomial basis is used. 
Expressing the data values for $N_{\boldsymbol{x}, n}$ by the signal vector

$$
\boldsymbol{f}=\boldsymbol{f}\left(N_{\boldsymbol{x}, n}\right)=\left(f\left(\boldsymbol{x}_{N, 1}\right), f\left(\boldsymbol{x}_{N, 2}\right), \ldots, f\left(\boldsymbol{x}_{N, n^{2}}\right)\right)^{T} \in \mathbb{R}^{n^{2}}
$$

and letting $\left\{\boldsymbol{b}_{1}, \boldsymbol{b}_{2}, \ldots, \boldsymbol{b}_{m}\right\}$ constitute a set of $m<n^{2}$ linearly independent bases spanning a subspace $S$ of $\mathbb{R}^{n^{2}}$, it is possible to approximate $\boldsymbol{f}$ by its projection $\boldsymbol{f}_{S}$ onto $S$. By letting $\boldsymbol{B}=\left(\boldsymbol{b}_{1}, \boldsymbol{b}_{2}, \ldots, \boldsymbol{b}_{m}\right)^{T}$ denote the basis matrix and $\boldsymbol{c}=\left\{c_{1}, c_{2}, \ldots, c_{m}\right\}$ represent the corresponding coefficients for $\boldsymbol{f}_{S}$, we can write $\boldsymbol{f}_{S}=\boldsymbol{B} \boldsymbol{c}$. The coefficients contained in $\boldsymbol{c}$ are given by

$$
\arg \min _{\boldsymbol{c} \in \mathbb{R}^{m}}\left\|\boldsymbol{f}_{S}-\boldsymbol{f}\right\|=\arg \min _{\boldsymbol{c} \in \mathbb{R}^{m}}\|\boldsymbol{B} \boldsymbol{c}-\boldsymbol{f}\|,
$$

which can be recognized as a least squares problem.

However, an adjustment of the influences of the different pixels in $N_{\boldsymbol{x}, n}$ is desired so that pixels closer to $\boldsymbol{x}$ have a greater impact on the result than those further away. Care should also be taken to the reliability of the values in the neighborhood pixels. These desired objectives of pixelwise influence and reliability can be achieved by using normalized convolution with the diagonal matrices for applicability and certainty given by

$$
\boldsymbol{W}_{\boldsymbol{a}}=\left(\begin{array}{ccc}
w_{a}\left(\boldsymbol{x}_{N, 1}\right) & \cdots & 0 \\
\vdots & \ddots & \vdots \\
0 & 0 & w_{a}\left(\boldsymbol{x}_{N, n^{2}}\right)
\end{array}\right) \text { and } \boldsymbol{W}_{\boldsymbol{c}}=\left(\begin{array}{ccc}
w_{c}\left(\boldsymbol{x}_{N, 1}\right) & \cdots & 0 \\
\vdots & \ddots & \vdots \\
0 & 0 & w_{c}\left(\boldsymbol{x}_{N, n^{2}}\right)
\end{array}\right)
$$

respectively. For all pixels in the neighborhood $N_{\boldsymbol{x}, n}, w_{a}\left(\boldsymbol{x}_{N, k}\right)$ is a Gaussian mask providing applicability weights and $w_{c}\left(\boldsymbol{x}_{N, k}\right)$ is the corresponding confidence mask, where $k=1,2, \ldots, n^{2}$. Following the outline provided by Farnebäck [4, influences of the neighborhood pixels in (4) are assigned weights by a matrix $\boldsymbol{W}$, implicitly defined by $\boldsymbol{W}^{2}=\boldsymbol{W}_{a} \boldsymbol{W}_{c}$ (Fig. 1). A vector $\boldsymbol{c}_{\boldsymbol{W}}$, representing the basis coefficients for the weighted neighborhood, can then be obtained from

$$
\arg \min _{\boldsymbol{c}_{\boldsymbol{W}} \in \mathbb{R}^{m}}\|\boldsymbol{W} \boldsymbol{B} \boldsymbol{c}-\boldsymbol{W} \boldsymbol{f}\| \text {. }
$$

The solution to this problem is then given by

$$
\boldsymbol{c}_{\boldsymbol{W}}=\left(\boldsymbol{B}^{T} \boldsymbol{W}_{a} \boldsymbol{W}_{c} \boldsymbol{B}\right)^{-1} \boldsymbol{B}^{T} \boldsymbol{W}_{a} \boldsymbol{W}_{c} \boldsymbol{f},
$$

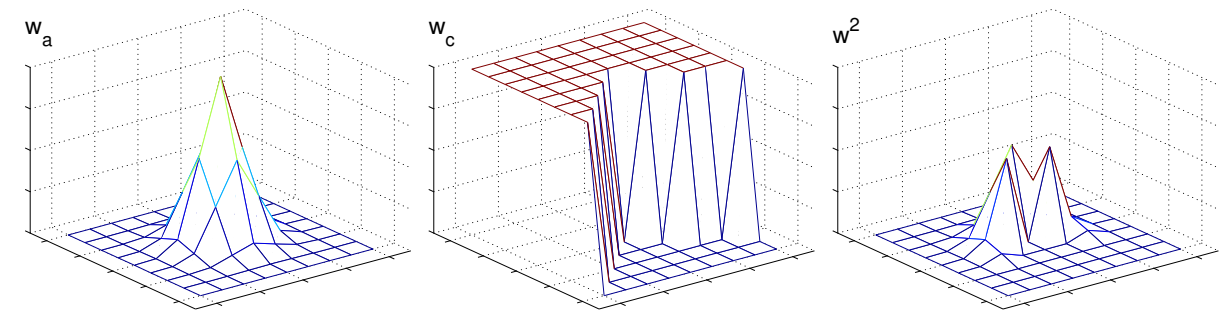

Fig. 1. Example of neighborhood weights corresponding to values of $W_{a}$ (left), $W_{c}$ (center) and $W^{2}$ (right) for $N_{\boldsymbol{x}, 9}$ 
which can be efficiently solved [4]. Once the coefficients in $\boldsymbol{c}_{\boldsymbol{W}}$ have been calculated, the coefficient corresponding to the constant base function can be retrieved, providing the approximation of the pixel value $f(\boldsymbol{x})$.

\section{Experiments and Results}

Three different data sets were reconstructed by both ordinary normalized convolution (NC) and the PINC algorithm:

1. A gray-scale image, corrupted by randomly removing $90 \%$ of the pixels.

2. The same image as in 1 , with holes of three different shapes.

3. 3D surface data for a pile of rocks, where data is partially missing.

\subsection{Reconstruction of Randomly Removed Data}

Figure 2 shows an original image and a version with $90 \%$ of the pixels randomly removed. Reconstructions were performed using a neighborhood size for coefficient extraction of $15 \times 15$ and a Gaussian mask with $\sigma=1.5$ as the applicability function. The original image is restricted to values in the range $\mathbb{R}[0,1]$, wherefore these limits were chosen as constraints for the reconstruction. Results of the NC and PINC algorithms, using zeroth and second order polynomials, are presented in Fig. 3. It should be noted that the PINC algorithm provides a less detailed result, but shows a more stable behavior for higher order polynomials where ordinary normalized convolution returns small regions of extreme values (Fig. 3, upper right).

\subsection{Reconstruction of Holes}

Figure 4 shows the same image as in the previous section (Sec. 3.1), now artificially corrupted by creating holes. The resulting reconstructed images, obtained by using the same parameter setup as in the previous section, are presented in Fig. 5. Differences between the two reconstruction algorithms are visible, especially in the row of circles crossing the face region. Black and white defects remain in the lower section of the reconstructions performed by ordinary normalized convolution, especially for higher order polynomial expansions.

\subsection{Reconstruction of Missing 3D Surface Data}

3D surface data from a structured lighting sensor [9] comprising a camera and a projector was used to test the algorithm. The data consists of spatially separated rows of $3 \mathrm{D}$ data points recorded on a $256 \times 256$ image grid and contains occlusions where the surface structure obscures the reflected light from reaching the camera (Fig. 6, right). The geometry of the sensor provides a pixelwise upper limit for the reconstruction of the occluded data, in the form of a linear interpolation between the measured pixels. 

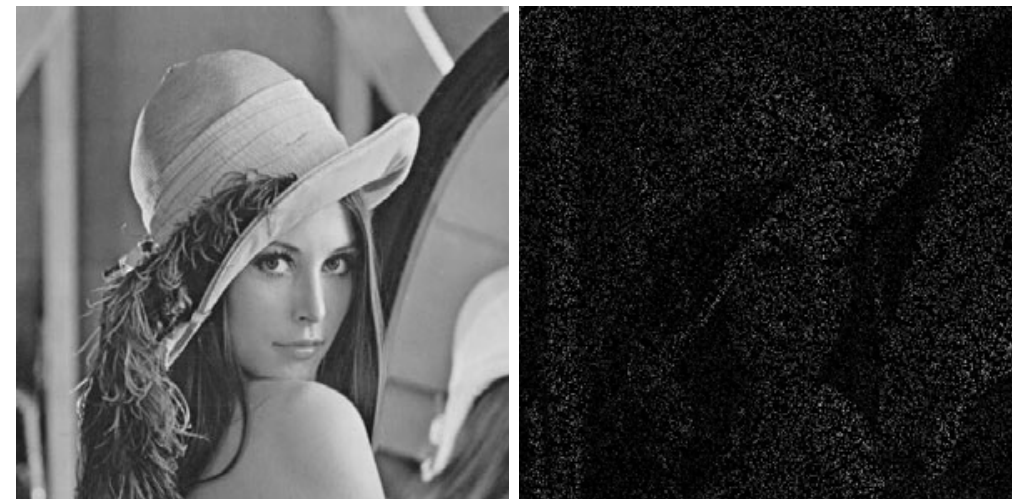

Fig. 2. The original image (left) and the version with $90 \%$ randomly removed data (right)
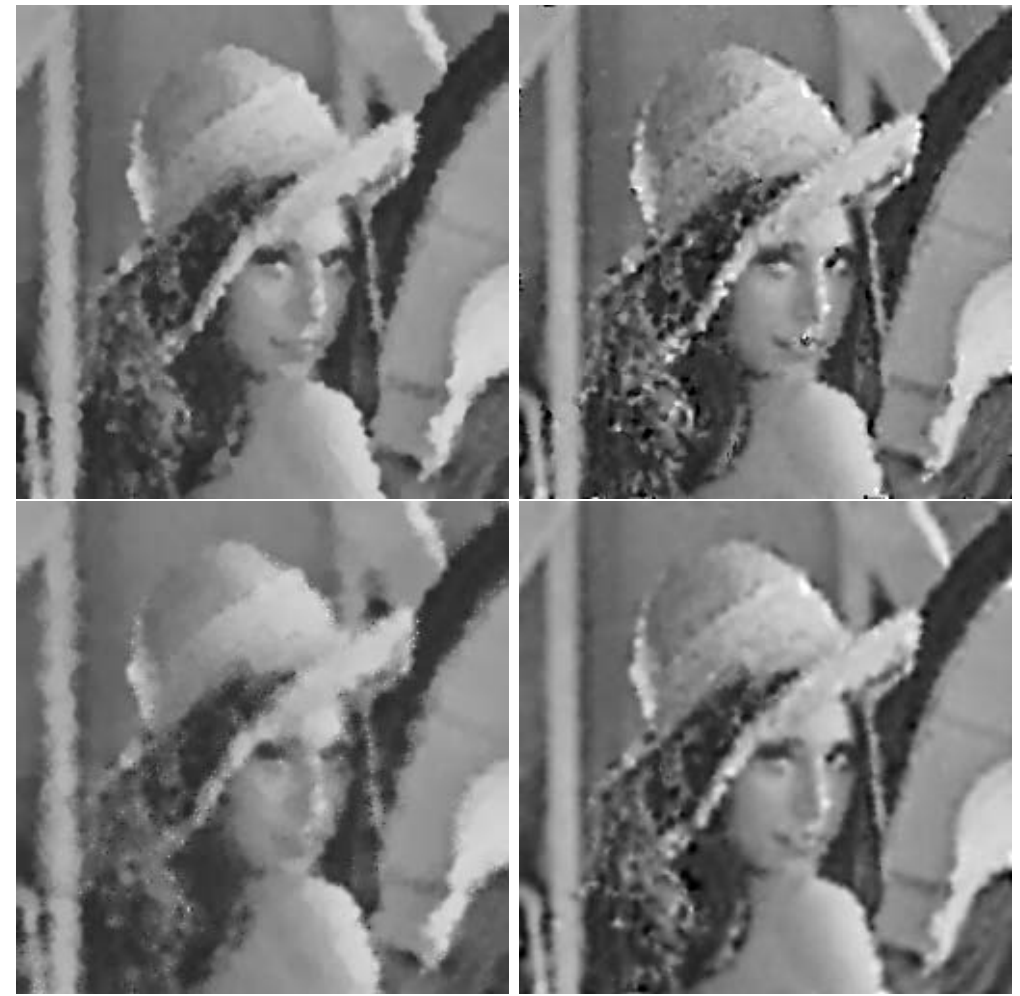

Fig. 3. Reconstructions of the right image in Fig. 2] using NC (first row) and PINC (second row). Order of polynomials used for interpolation are 0 (left) and 2 (right). 


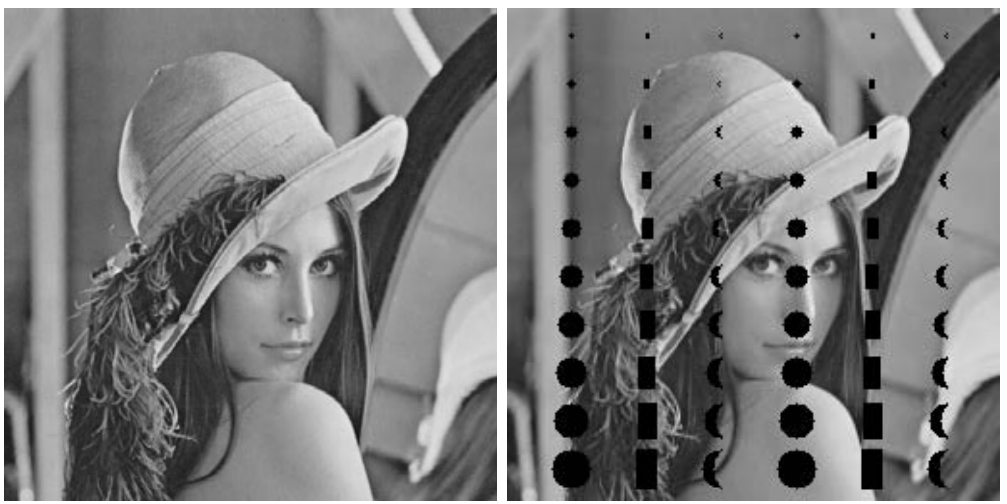

Fig. 4. The original image (left) and a version with holes (right)

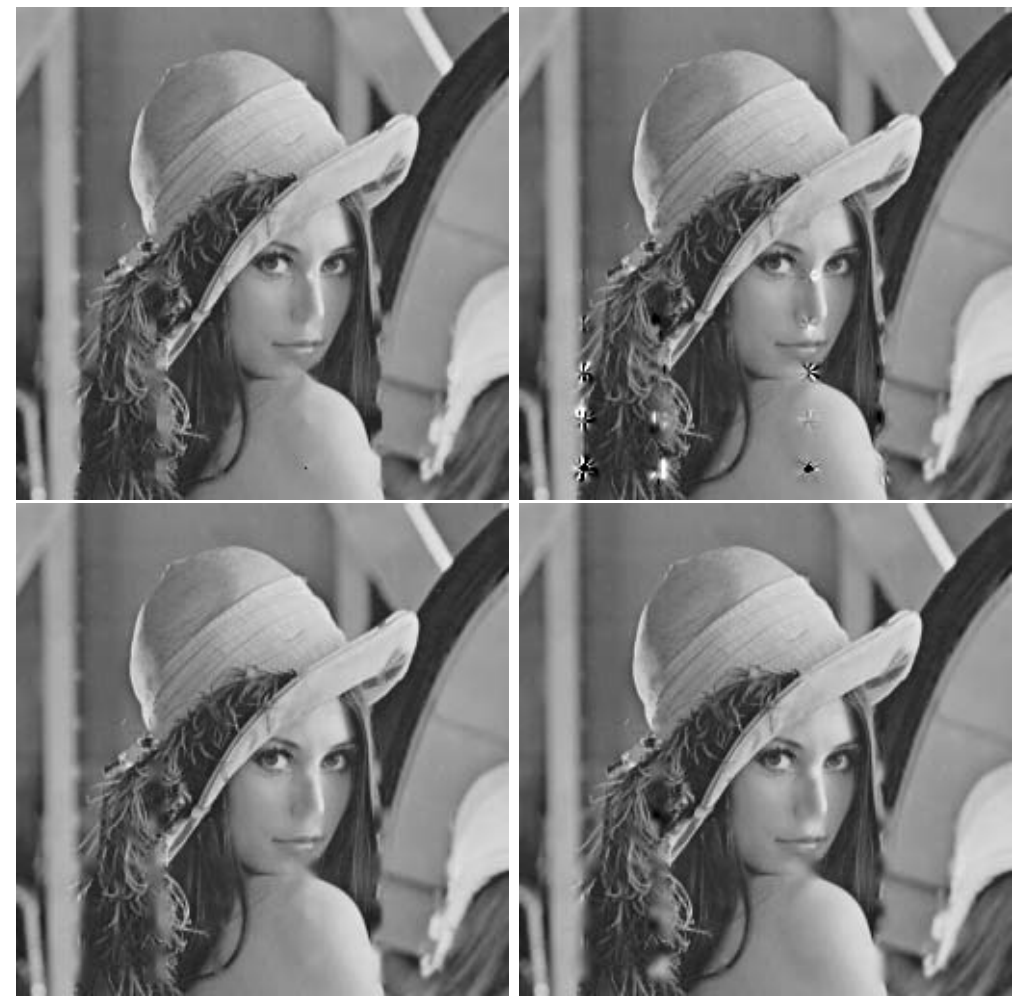

Fig. 5. Reconstructions of the right image in Fig. 4 using NC (first row) and PINC (second row). Order of polynomials used for interpolation are 0 (left) and 2 (right). 

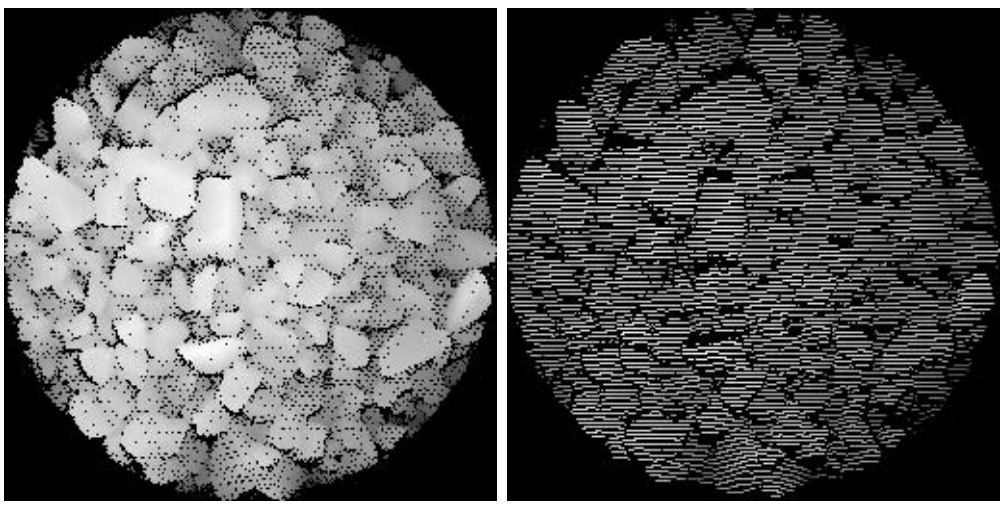

Fig. 6. 3D surface data of a rock pile: Combined information from all six measurements (left) and from one measurement only (right)
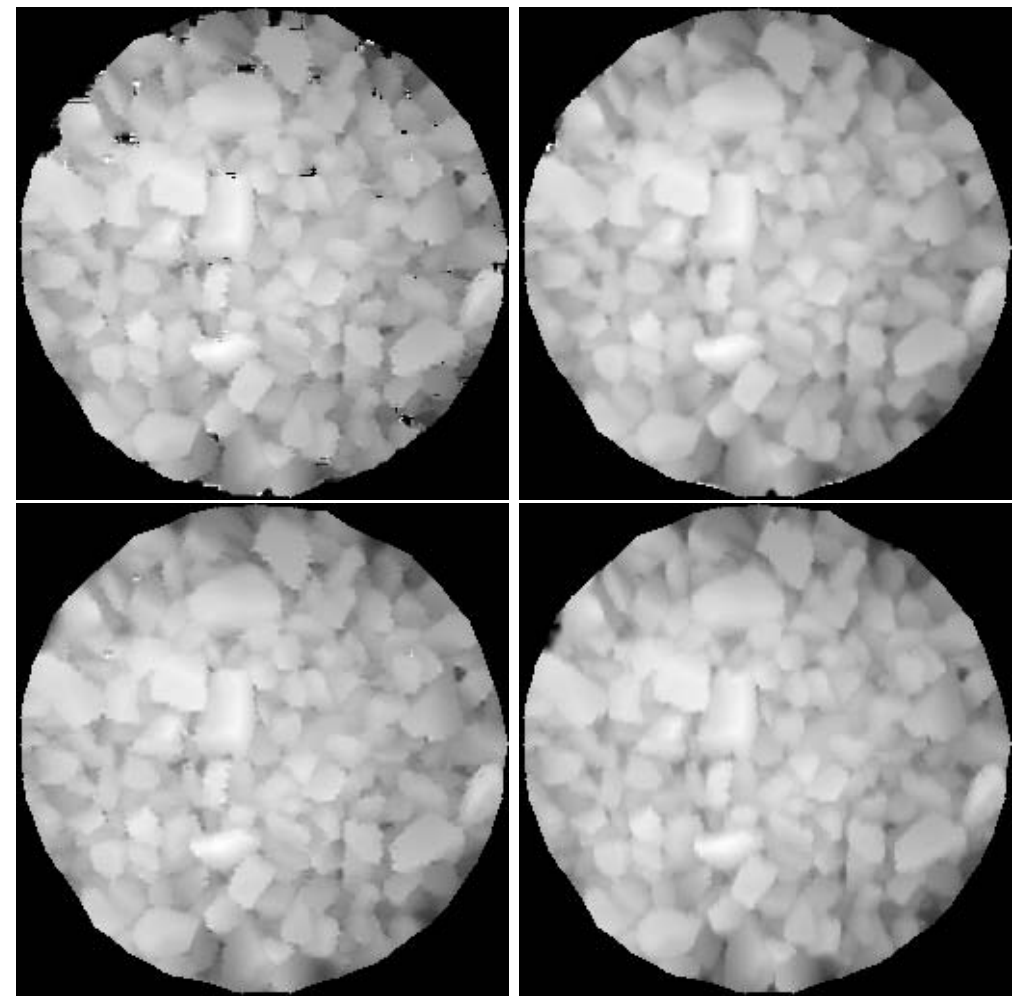

Fig. 7. Reconstructions of 3D surface data (Fig. 6, right) using $9 \times 9$ neighborhood $\mathrm{NC}$ (upper left), $15 \times 15$ neighborhood NC (upper right), adaptive neighborhood NC (lower left), and $9 \times 9$ neighborhood PINC (lower right). Order 2 polynomials were used, giving RMSE values $0.055,0.0310 .030$ and 0.029 respectively. 
The performance of the PINC algorithm was measured by calculating Root Mean Squared Error (RMSE) values between the interpolated data and a second data set comprising six overlapping measurements (Fig. 6, left), rescaling the data sets to gray scale images of range $\mathbb{R}[0,1]$. For comparison, the surface was also reconstructed by three different $\mathrm{NC}$ approaches. First, a neighborhood of the same size as used for the PINC algorithm, $9 \times 9$ pixels, was applied. Secondly, the neighborhood was extended to $15 \times 15$ to avoid the type of holes visible in the NC results presented in Fig. 5 (upper right). Thirdly, the NC algorithm was used with adaptive neighborhoods, where for each pixel the smallest surrounding neighborhood containing at least $25 \%$ valid data was used for interpolation. Results for second order polynomials are presented in Fig. 7. For the $9 \times 9$ neighborhood NC reconstruction, the RMSE value for the resulting data is 0.055 . It should be noted that in this case the small neighborhood does not bridge the regions of missing data, giving a potential large error for those pixels. The $15 \times 15$ neighborhood NC reconstruction fills in the holes, providing an RMSE value of 0.031. NC reconstruction using an adaptive neighborhood gives a lower RMSE value, 0.030. Finally, the suggested PINC algorithm gives the RMSE value 0.029.

\section{Discussion}

As can be seen in Fig. 3, the PINC algorithm does not reconstruct fine details as effectively as $\mathrm{NC}$ on $90 \%$ randomly distributed missing data. The reconstruction by growing property of PINC can result in image structure from a location with a local cluster of pixels, spreading over the image and influencing the reconstruction around more isolated pixels. However, while the presented incremental method is less likely to capture small details in the randomly sampled data, it is less sensitive to extreme values when using higher order polynomials.

From Fig. 5] it is clear that the suggested incremental approach fills in missing data where ordinary normalized convolution does not. This is because the chosen neighborhood is too small to bridge the largest holes. The problem can be approached by using adaptive neighborhoods, as described in 17, 8, Also, the result from the PINC algorithm is in general more pleasant to the eye than the $\mathrm{NC}$ reconstructed image.

Presented results for $3 \mathrm{D}$ range data shows that our method gives the best RMSE value for the tested data. Also, as expected, we see that $\mathrm{NC}$ needs a larger neighborhood to cover the missing regions. The use of locally adaptive neighborhoods partially solves this problem, but demands more computational power due to the unconstrained size of the neighborhoods when available pixels become very sparse. However, since the PINC algorithm currently reaches the same performance as $\mathrm{NC}$ with adaptive neighborhood sizes, it should be possible to improve PINC by incorporating the techniques that adapt to their surroundings such as neighborhood and applicability presented in [7, 8].

Even though the NC reconstruction with a $15 \times 15$ neighborhood here produces an RMSE value that is comparable to PINC and adaptive NC, this is not something we can expect to be true in the general case. The images used in 
this work are at quite low resolution and all occluded regions are roughly of the same magnitude which in this case makes the $15 \times 15$ neighborhood suitable for all regions. When having occluded regions of different size, this means that for using $\mathrm{NC}$ we would have to use neighborhoods that bridges the largest occluded region, something that would introduce smoothing in the smaller cavities.

\section{Conclusion}

By measuring RMSE values between a reconstructed partially occluded 3D rock pile surface and its true topology, we conclude that the suggested image reconstruction by prioritized incremental normalized convolution (PINC) performs better than ordinary normalized convolution (NC). To adapt the size of neighborhoods seem to be another possible approach of improving the performance of $\mathrm{NC}$, but we have shown that a comparable result can be achieved using smaller neighborhoods.

The presented hole filling and reconstruction of randomly sampled data (Figs 5 and 3. respectively) highlights the differences between PINC and NC algorithms. The PINC algorithm is not adapted for reconstruction of data sets where most data is randomly removed, but is useful for its purpose; filling holes in 3D surface data.

\section{References}

[1] Averbuch, A., Gelles, G., Schclar, A.: Fast hole-filling in images via fast comparison of incomplete patches. In: Gunsel, B., Jain, A.K., Tekalp, A.M., Sankur, B. (eds.) MRCS 2006. LNCS, vol. 4105, pp. 738-744. Springer, Heidelberg (2006)

[2] Bertalmio, M., Sapiro, G., Caselles, V., Ballester, C.: Image inpainting. In: Proceedings of the 27th Annual Conference on Computer Graphics and Interactive Techniques, SIGGRAPH 2000, pp. 417-424. ACM Press, New York (2000)

[3] Faille, F., Petrou, M.: Invariant image reconstruction from irregular samples and hexagonal grid splines. Image and Vision Computing 28(8), 1173-1183 (2010)

[4] Farnebäck, G.: Polynomial Expansion for Orientation and Motion Estimation. PhD thesis, Linköping University, Sweden (2002)

[5] Ju, T.: Fixing geometric errors on polygonal models: A survey. Journal of Computer Science and Technology 24, 19-29 (2009)

[6] Knutsson, H., Westin, C.-F.: Normalized and differential convolution. In: Proceedings of IEEE Computer Society Conference on Computer Vision and Pattern Recognition, CVPR 1993, pp. 515-523 (June 1993)

[7] Pham, T.Q., van Vliet, L.J., Schutte, K.: Robust fusion of irregularly sampled data using adaptive normalized convolution. EURASIP J. Appl. Signal Process. 2006, 236-236 (2006)

[8] Takeda, H., Farsiu, S., Milanfar, P.: Kernel regression for image processing and reconstruction. IEEE Transactions on Image Processing 16(2), 349-366 (2007)

[9] Thurley, M.J., Ng, K.C.: Identifying, visualizing, and comparing regions in irregularly spaced 3D surface data. Computer Vision and Image Understanding 98(2), 239-270 (2005) 